\title{
Description of the Connected Components of a Semialgebraic Set in Single Exponential Time*
}

\author{
Joos Heintz, ${ }^{1}$ Marie-Francoise Roy, ${ }^{2}$ and Pablo Solernó ${ }^{1}$ \\ ${ }^{1}$ Departamento de Matemática, Facultad de Ciencias Exactas y Naturales, \\ Universidad de Buenos Aires, Ciudad Universitaria. Pabellón I, \\ (1428) Buenos Aires, Argentina \\ joos@mate.edu.ar \\ ${ }^{2}$ IRMAR, Université de Rennes I, \\ 35042 Rennes Cedex, France \\ costeroy@univ-rennes1.fr
}

To our friend Mario Raimondo

\begin{abstract}
This paper is devoted to the following result: let $\mathbf{R}$ be a real closed field and let $S$ be a semialgebraic subset of $\mathbf{R}^{n}$ defined by a boolean combination of polynomial inequalities. Let $D$ be the sum of the degrees of the polynomials involved. Then it is possible to find algorithmically a description of the semialgebraically connected components of $S$ in sequential time $D^{n^{(01)}}$ and parallel time $(n \log D)^{O(1)}$. This implies that the problem of finding the connected components of a semialgebraic set can be solved in P-SPACE.
\end{abstract}

\section{Introduction}

\subsection{The Statement}

We denote by $\mathbf{R}$ a real closed field, by $\mathbf{A}$ a subring of $\mathbf{R}$, and by $S$ a semialgebraic (over $\mathbf{A}$ ) subset of $\mathbf{R}^{n}$. Let $X_{1}, \ldots, X_{n}$ be indeterminates over $\mathbf{R}$. We suppose that $S$ is given by a boolean combination of polynomial inequalities involving polynomials $F_{1}, \ldots, F_{s}$ of $\mathbf{A}\left[X_{1}, \ldots, X_{n}\right]$. We consider $F_{1}, \ldots, F_{s}$ as the standard input

\footnotetext{
* This work was partially supported by POSSO BRA 6846 .
} 
of all algorithms we design in what follows. The length of this input is determined by the parameters $n, D:=\sum_{1 \leq j \leq s} \operatorname{deg} F_{j}$, and, in the case of $\mathbf{A}:=\mathbb{Z}$, by the maximal binary length $\sigma$ of the coefficients of $F_{1}, \ldots, F_{s}$. We think of the polynomials $F_{1}, \ldots, F_{s}$ as represented in dense form by their coefficient vectors, Thus the size of our input is $O\left(s D^{n}\right)$ if we are working in an algebraic complexity model and $O\left(s \sigma D^{n}\right)$ if we are working in the bit-model and if $\mathbf{A}:=\mathbb{Z}$.

The notion of algorithm which we use is that of a uniform family of arithmetical networks over A parametrized by $D$ and $n$ (see [G1] for definitions). By sequential and parallel complexity of the algorithm (or sequential and parallel time) we refer to the size and the depth of the arithmetic networks representing the algorithm. We call an algorithm admissible (or single exponential) if its sequential execution time is $D^{n^{O(1)}}$ and its parallel execution time is $(n \log D)^{O(1)}$.

In case $\mathbf{A}:=\mathbb{Z}$ all our (admissible) algorithms can be simulated by uniform families of boolean networks of size $D^{n^{O(1)}} \sigma^{O(1)}$ and depth $(n \log D \sigma)^{O(1)}$. Therefore they can be executed in deterministic Turing machine time $D^{n^{o(1)}} \sigma^{o(1)}$ and Turing machine space $(n \log D \sigma)^{O(1)}$.

Since these simulation arguments are absolutely straightforward we restrici ourselves to the (algebraic) complexity model of arithmetical networks over $\mathbf{A}$.

The central result of this paper is the following:

Main Theorem. It is possible to compute by a uniform family of arithmetical networks of size $D^{n^{o(1)}}$ and depth $(n \log D)^{O(1)}$ (i.e., in admissible time) a description of each semialgebraically connected component of $S$. This description is given by $a$ set of $D^{O(n)}$ quantifier-free formulas, each defining a semialgebraically connected component of $S$. The number and the degree of the polynomials (of $\mathbf{A}\left[X_{1}, \ldots, X_{n}\right]$ ) involved in these formulas is of order $D^{n^{\text {o(1) }}}$.

\subsection{Comments}

Our main result follows the line of various papers devoted to the complexity of algorithms in real algebraic geometry (and topology) in single exponential time [GV1], [GV2], [G2], [Ca1], [Ca2], [R1]-[R3], [GHR $\left.{ }^{+}\right],[\mathrm{GR}],[\mathrm{HRS} 1]-$ [HRS5].

A similar algorithmic result with a sequential complexity bound which is doubly exponential in $n$ was already known (see, for example, [Co] or [SS]). The doubly exponential behavior of the complexity in these papers is due to the use of iterated projections in the process of the so-called cylindric algebraic decomposition on which they are based.

The first result with single exponential complexity bounds concerning the topology of semialgebraic sets may be found in [Ca2], where the essential geometric idea, which we also use, consists of constructing continuous curves on the semialgebraic set $S$ directly instead of doing this by means of iterated projections. There are nevertheless significant differences between Canny's results and ours. Namely, we have no restrictive hypothesis (such as general position) on 
the semialgebraic set. We do not need a Whitney stratified input nor do we make use of the theory of stratifications.

This paper is a direct continuation of the articles [HRS3] and [HRS4], which treat the problem of single exponential path and roadmap finding in semialgebraic sets. (See also [GV2] and [GR] for analogous results concerning this question and $\left[\mathrm{GHR}^{+}\right]$for an expository presentation of the methods used.) Our main theorem was announced in [HRS5] and the corresponding result for the sequential bit complexity model was obtained in [CGV].

We use an approach which is somewhat different from [GV2] and [CGV] which we developed independently (and simultaneously). We hope that our approach contributes a clarification of the complicated algorithmic structure of the subject.

We use some notions and results from differential topology and the topology of semialgebraic sets. We also need some ideas from real algebraic geometry, the notion of semialgebraically connected components of semialgebraic sets over an arbitrary real closed field (see [BCR]) for example, or the use of infinitely small (or large) elements and Puiseux series (see [GV1], [G2], and [HRS2]-[HRS4]). We employ the "efficient" quantifier-elimination method of [HRS2] (or of [R3]) in various situations such as the computation of the closure and the interior of a semialgebraic set and the computation of the image of a semialgebraic function.

\subsection{Definitions and Notations}

Let $x:=\left(x_{1}, \ldots, x_{n}\right)$ and $y:=\left(y_{1}, \ldots, y_{n}\right)$ be two points of $\mathbf{R}^{n}$. We write

$$
|x-y|:=\sqrt{\left(x_{1}-y_{1}\right)^{2}+\cdots+\left(x_{n}-y_{n}\right)^{2}}
$$

for their euclidean distance. If $r$ is a positive element of $\mathbf{R}$ we write

$$
B(x, r):=\left\{y \in \mathbf{R}^{n} ;|x-y|<r\right\}
$$

for the open ball of radius $r$ centered at $x$.

We think of the (real) affine space $\mathbf{R}^{n}$ as being equipped with the euclidean topology and the semialgebraic subsets of $\mathbf{R}^{n}$ with the induced one (which we call euclidean as well).

For a given subset $M \subset \mathbf{R}^{n}$ we write $\bar{M}$ for its closure in the euclidean topology.

We denote by $\mathscr{L}(\mathbf{A})$ the elementary language of ordered fields with constants from $\mathbf{A}$. Terms of $\mathscr{L}(\mathbf{A})$ are considered as polynomials with coefficients in $\mathbf{A}$. (See Section 2.2 of [BCR].)

The Tarski-Seidenberg Principle states that the sets definable by elementary (first-order) formulas from $\mathscr{L}(\mathbf{A})$ are exactly the semialgebraic sets over $\mathbf{A}$.

For a formula $\Phi \in \mathscr{L}(\mathbf{A})$ we write $d(\Phi)$ for the sum of the (total) degrees of the polynomials appearing as terms in $\Phi$ and call this quantity the degree of $\Phi$. 
We say that a semialgebraic subset $S$ of $\mathbf{R}^{n}$ is explicitly given if a boolean combination of polynomial inequalities (or equivalently a quantifier-free formula $\Phi$ of $\mathscr{L}(\mathbf{A})$ ) defining $S$ is given. In this case we use the (somewhat lax) notation $d(S)$ instead of $d(\Phi)$.

Let $S \subset \mathbf{R}^{n}$ be an explicitly given semialgebraic set with $D:=d(S)$. We say that an algorithm which accepts the polynomials defining $S$ is admissible if it runs in sequential time $D^{n^{(1)}}$ and in parallel time $(n \log D)^{O(1)}$.

\section{The Strategy of the Proof}

\subsection{The Case of a Bounded Smooth Hypersurface}

Let us suppose from now on that $S$ is a bounded and smooth semialgebraic hypersurface of $\mathbf{R}^{n}$ defined as the zeros of a polynomial $F \in \mathbf{A}\left[X_{1}, \ldots, X_{n}\right]$ which has the property that $\nabla F:=\left(\partial F / \partial X_{1}\right)^{2}+\cdots+\left(\partial F / \partial X_{n}\right)^{2}$ vanishes nowhere on $S$. (We call such a polynomial $F$ a regular equation of the bounded hypersurface $S$ ). We denote by $D:=\operatorname{deg} F$ the degree of $F$.

Our first goal is the construction of families of semialgebraic curves which describe in a certain manner the semialgebraically connected components of $S$. For this purpose we introduce the following notion of a roadmap (see also [Ca2]):

Definition 2.1. Let $W \subset \mathbf{R}^{n}$ be a semialgebraic set. We call a semialgebraic set $\Re \subset W$ a roadmap for $W$ if $\operatorname{dim} \Re \leq 1$ and if the intersection of any semialgebraically connected component of $W$ with $\Re$ is nonempty and semialgebraically connected. (Here dim $\mathfrak{R}$ denotes the dimension of the semialgebraic set $\mathfrak{R}$. See Section 2.8 of $[\mathrm{BCR}])$.

In [HRS3] and [HRS4] we designed an admissible algorithm for the construction of a suitable roadmap in an arbitrary semialgebraic set $W$. This algorithm also furnishes an admissible procedure to join any semialgebraically definable point of $W$ (i.e., any point of $W$ with algebraic coordinates over A) with the roadmap by means of a semialgebraically connected curve in $W$. We show in Sections 3 and 4 that this join procedure can be realized in a uniform way in the hypersurface $S$. Thus we obtain an admissible algorithm which allows us to connect any point of $S$ (not only the semialgebraically definable ones) with a given roadmap of $S$ (see Lemma 11 below).

Let $1 \leq i<n$. We write $\pi_{i}: S \rightarrow \mathbf{R}$ for the semialgebraic projection (or coordinate) function induced by the variable $X_{i}$ on $S$. For any point $x:=\left(x_{i+1}, \ldots, x_{n}\right)$ of $\mathbf{R}^{n-i}$ let $S_{x}:=\left\{\left(z_{1}, \ldots, z_{n}\right) \in S ; z_{i+1}=x_{i+1}, \ldots, z_{n}=x_{n}\right\}$ be the fiber of $x$ in $S$ and let $\pi_{i}^{(x)}: S_{x} \rightarrow \mathbf{R}$ be the restriction of $\pi_{i}$ to $S_{x}$. The fiber $S_{x}$ is a semialgebraic, closed, and bounded subset of $\mathbf{R}^{n}$ defined by the equation $F_{x}=0$, where $F_{x}:=$ $F\left(X_{1}, \ldots, X_{i}, x_{i+1}, \ldots, x_{n}\right)$ is a polynomial of $\mathbf{R}\left[X_{1}, \ldots, X_{i}\right]$.

We say that the fiber $S_{x}$ is smooth if $F_{x}$ is a regular equation of $S_{x}$.

Let us summarize the roadmap construction of [HRS3] (compare also $\left[\mathrm{GHR}^{+}\right]$) for the input polynomial $F$ : 
The whole algorithm is based on two admissible procedures which both produce semialgebraically connected curves in $S$ together with their endpoints:

(a) The first one consists of constructing a finite semialgebraic partition of the $X_{n}$-axis and in determining, for each piece of this partition (represented as a closed interval), a finite number of continuous semialgebraic functions having the following properties:

The graph of each of these functions is contained in $S$ and thus defines a semialgebraically connected curve in $S$.

The curves which are given by these continuous semialgebraic functions on a fixed interval meet every semialgebraically connected component of each $\pi_{n}$-fiber along the interval (see Corollary 2 of [HRS3] or Theorem 2 of $\left[\mathrm{GHR}^{+}\right]$.

(b) The second procedure is an admissible version of the Curve Selection Lemma in real algebraic geometry [HRS3, Corollary 4]. It consists of constructing connected curves starting from any given point $x:=\left(x_{1}, \ldots, x_{n}\right)$ which lies, e.g., in the fiber $S_{x_{n}}$ of a critical value $x_{n}$ of the function $\pi_{n}$. These curves enter every semialgebraically connected component of the intersection of a suitable open ball around $x$ with the fibers $S_{x_{n}+t}$ and $S_{x_{n}-t}$ for sufficiently small $t>0$.

These curves are used in the roadmap construction to move fibers of critical values of $\pi_{n}$ into fibers of noncritical (regular) values.

By means of procedures (a) and (b), in [HRS3] we designed an admissible algorithm for a roadmap construction in $S$ as follows (our algorithm is recursive in $n$ and starts from the input polynomial $F$ ):

(1) In admissible time an $\mathbf{A}$-linear transformation of the variables $X_{1}, \ldots, X_{n}$ is constructed which induces on $S$ an $M$-function $\pi: S \rightarrow \mathbf{R}$ (following the terminology of [HRS3] we call a Nash function an $M$-function if it has only finitely many critical points). Let us assume without loss of generality that $\pi=\pi_{n}$.

(2) Procedure (a) is applied to the $X_{n}$-axis in order to obtain a family of connected curves which intersect all semialgebraically connected components of all $\pi_{n}$-fibers (see Section 4, Step 2 of [HRS3]).

(3) For each critical point of $\pi_{n}$ and each point which arises by intersecting any curve constructed in (2) with the fiber of a critical value of $\pi_{n}$, we produce by procedure (b) a set of connected curves starting from that point.

(4) Subdividing the $X_{n}$-axis, once more if necessary, into closed semialgebraic intervals we assure that each curve constructed in (2) and (3) contains at most one point (which is an endpoint) lying in a fiber of a critical value of $\pi_{n}$.

Let $\mathscr{K}$ be the set of curves constructed in (2) and (3) and subject to the rearrangement (4) and let $\mathscr{C} \subset S$ be the set of the endpoints of the curves contained in $\mathscr{K}$. 
(5) For each $x_{n} \in \pi_{n}(\mathscr{C})$ which is not a critical value of $\pi_{n}$ (i.e., the fiber $S_{x_{n}}$ is smooth) we link all the points of $S_{x_{n}} \cap \mathscr{C}$ which lie in the same semialgebraically connected component of $S_{x_{n}}$ by means of a connected semialgebraic curve produced by a recursive call of our algorithm for the input polynomial $F\left(X_{1}, \ldots, X_{n-1}, x_{n}\right)$ which is a regular equation for the smooth bounded hypersurface $S_{x_{n}}$ of the $(n-1)$-dimensional real affine space $\mathbf{R}^{n-1} \times\left\{x_{n}\right\}$.

In this paper Propositions 1 and 5 below are parametric versions of procedures (a) and (b). We construct in advance the semialgebraic curves which the above algorithm produces by its recursive calls of (2) and (3). The recursive argument of (4) is replaced by a limited number of applications of the effective quantifierelimination algorithm for real closed fields [HRS2] (or [R3]) to certain prenex formulas which contain only one block of (existential) quantifiers. These formulas represent the result of the composition of at most $n$ semialgebraic maps (see Lemma 11 and Remark 12).

Finally, the recursive application of (1), where we construct a suitable $A$-linear transformation of the variables in order to obtain coordinates which represent $M$-functions, is replaced in the next section by a uniform choice of $M$-projections which can be done in advance (see Remark 10).

\subsection{The General Case}

Using infinitesimal deformations and quantifier elimination for computing limits, it is now possible to compute in admissible time the connected components of any semialgebraic set from the connected components of a smooth bounded hypersurface, according to Sections 2 and 3 of [CGV] or Section 4 of [HRS4].

\section{Parametric Roadmaps}

We denote by $p_{i}$ the canonical projection: $p_{i}: \mathbf{R}^{n} \rightarrow \mathbf{R}^{i}$ defined by

$$
p_{i}\left(x_{1}, \ldots, x_{n}\right):=\left(x_{1}, \ldots, x_{i}\right) \quad \text { for } \quad\left(x_{1}, \ldots, x_{n}\right) \in \mathbf{R}^{n}
$$

For a given set $W \subset \mathbf{R}^{n}$ and a point $x^{\prime} \in \mathbf{R}^{i}$, we write $W_{x^{\prime}}:=p_{i}^{-1}\left(x^{\prime}\right) \cap W$.

In the next proposition we describe the fibers of a projection of a given semialgebraic set by means of a parametrized family of continuous semialgebraic curves of admissible complexity.

Proposition 1. Let $W \subset \mathbf{R}^{n}$ be an explicitly given bounded, closed semialgebraic set. Given a fixed index $i, 1 \leq i<n$, it is possible to construct in admissible time a semialgebraic subdivision $\left(\mathscr{U}_{l}\right)_{1 \leq l \leq N}$ of $p_{i}(W)$, a semialgebraic subdivision $\left(\mathscr{T}_{l}\right)_{1 \leq l \leq N}$ 
of $p_{i+1}(W)$, and a family $\left(\eta_{l j}\right)_{1 \leq l \leq N, 1 \leq j \leq N_{l}}$ of continuous semialgebraic functions $\eta_{l j}: \mathscr{T}_{l} \rightarrow \mathbf{R}^{n-i-1}$ such that the following conditions are satisfied:

(i) For any pair of indices $(l, j)$, the graph of $\eta_{(l, j)}$ is contained in $W$.

(ii) For any index $l$ and any point $z \in \mathscr{U}_{l}$, the fiber $\left(\mathscr{T}_{l}\right)_{z}$ is a closed subinterval of the line $\left(\mathbf{R}^{i-1}\right)_{z}$.

(iii) For any point $x^{\prime} \in p_{i+1}(W)$ and for any semialgebraically connected component of $W_{x^{\prime}}$, a pair of indices $(l, j)$ exist such that $\eta_{(l, j)}\left(x^{\prime}\right)$ belongs to this component.

Proof. The fact that $W$ is closed and bounded implies that $p_{i}(W)$ and $p_{i+1}(W)$ are closed and bounded too.

Applying Theorem 7 of [HRS2] (compare also Theorem 1 of [HRS3]) to the semialgebraic projection induced by $p_{i+1}$ we find in admissible time a semialgebraic partition $\left(T_{m}\right)_{1 \leq m \leq M}$ of $p_{i+1}(W)$ and a family $\left(\xi_{(m, s)}\right)_{1 \leq m \leq M, 1 \leq s \leq M_{m}}$ of continuous semialgebraic functions $\xi_{(m, s)}: T_{m} \rightarrow \mathbf{R}^{n-i-1}$ with the following properties:

- For any pair of indices $(m, s)$, the graph of $\xi_{(m, s)}$ is contained in $W$.

- For any point $x^{\prime} \in p_{i+1}(W)$ and any semialgebraically connected component of $W_{x^{\prime}}$, a pair of indices $(m, s)$ exists such that $\xi_{(m, s)}\left(x^{\prime}\right)$ belongs to this component.

Furthermore, we may assume without loss of generality that the sets $T_{m}$ are defined by an admissible family of polynomials which is stable under derivation with respect to the variable $X_{i+1}$.

Since the semialgebraic sets $W$ and $p_{i+1}(W)$ are closed and bounded the sets $\bar{T}_{m}$ have the same property and they form a covering of $p_{i+1}(W)$. Moreover, we may extend the maps $\xi_{(m, s)}$ in admissible time to continuous semialgebraic functions $\xi_{(m, s)}: \bar{T}_{m} \rightarrow \mathbf{R}^{n-i-1}$ by means of the quantifier-elimination procedures of [HRS2] or [R3].

We subdivide in admissible time the family $\left(\bar{T}_{m}\right)_{1 \leq m \leq M}$ into a suitable partition $\left(\mathscr{T}_{l}\right)_{1 \leq l \leq N}$ of $p_{i+1}(W)$. The continuous semialgebraic functions $\eta_{(l, j)}: T_{l} \rightarrow \mathbf{R}^{n-i-1}$ are obtained by restricting the functions $\xi_{(m, s)}$. Therefore conditions (i) and (iii) of the proposition are automatically satisfied.

Since the sets $T_{m}$ are bounded and defined by a family of polynomials which is stable under derivations with respect to the variable $X_{i+1}$ we conclude by Thom's Lemma [BCR, Proposition 2.5.4] that, for any $z \in \mathbf{R}^{i}$, the fiber $\left(\bar{T}_{m}\right)_{z}$ is a bounded and closed subinterval of the line $\left(\mathbf{R}^{i+1}\right)_{z}$.

For an arbitrary index $m$ let us consider the semialgebraic subset $\tilde{T}_{m}$ of $\bar{T}_{m}$ defined by the formula:

$$
\begin{aligned}
\left(x_{1}, \ldots, x_{i+1}\right) \in \tilde{T}_{m} \Leftrightarrow(\forall \varepsilon)(\exists t) & (\varepsilon>0 \rightarrow((-\varepsilon<t<\varepsilon) \\
& \left.\left.\wedge\left(x_{1}, \ldots, x_{i}, x_{i+1}+t\right) \notin \bar{T}_{m} \wedge\left(x_{1}, \ldots, x_{i+1}\right) \in \bar{T}_{m}\right)\right) .
\end{aligned}
$$

We observe that $\tilde{T}_{m}$ is the set of extrema of all the intervals $\left(\bar{T}_{m}\right)_{z}$ where $z \in \mathbf{R}^{i}$.

It is possible to compute $\tilde{T}_{m}$ explicitly in admissible time by means of the quantifier-elimination procedures of [HRS2] or [R3]. 
Let $\pi: \mathbf{R}^{i+1} \rightarrow \mathbf{R}^{i}$ be the projection which forgets the $(i+1)$ th coordinate.

Since the semialgebraic sets $\bar{T}_{m}$ are closed and bounded, $\pi\left(\widetilde{T}_{m}\right)=\pi\left(\tilde{T}_{m}\right)$ is immediately verified.

Applying Theorem 7 of [HRS2] to the semialgebraic map $\widetilde{T}_{m} \rightarrow \mathbf{R}^{i}$ induced by the projection $\pi$ we obtain in admissible time for each $m$ a semialgebraic partition $\left(\tilde{\mathscr{U}}_{(m, r)}\right)_{1 \leq m \leq M, 1 \leq r \leq M_{m}}$ of $\pi\left(\tilde{T}_{m}\right)$ and, for each pair of indices $(m, r)$, two continuous semialgebraic functions $\alpha_{(m, r)}^{-}, \alpha_{(m, r)}^{+}: \tilde{\mathscr{U}}_{(m, r)} \rightarrow \mathbf{R}$ (representing opposite interval ends) satisfying the conditions:

- For any index $r$, the graph of $\alpha_{(m, r)}^{-}$and $\alpha_{(m, r)}^{+}$is contained in $\tilde{T}_{m}$ and the inequality $\alpha_{(m, r)}^{-}(z) \leq \alpha_{(m, r)}^{+}(z)$ holds for any $z \in \mathscr{U}_{(m, r)}$.

- For any point $z \in \mathbf{R}^{i}$ and for any semialgebraically connected component of $\left(\tilde{T}_{m}\right)_{z}$ there exists an index $r$ such that $\left(z, \alpha_{(m, r)}^{-}(z)\right)$ and $\left(z, \alpha_{(m, r)}^{+}(z)\right)$ belong to that component.

For given $m$ the semialgebraic sets $\tilde{\mathscr{U}}_{(m, r)}$ form a partition of $\pi\left(\tilde{T}_{m}\right)=\pi\left(\bar{T}_{m}\right)$. The sets $\bar{T}_{m}$ cover the image $p_{i+1}(W)$ and we have $p_{i}=\pi \circ p_{i+1}$. Therefore the sets $\tilde{\mathscr{U}}_{(m, r)}, 1 \leq m \leq M, 1 \leq r \leq M_{m}$, cover the semialgebraic set $p_{i}(W)$.

For the sake of notational simplicity let us rename the semialgebraic sets $\tilde{\mathscr{U}}_{(m, r)}$ and the functions $\alpha_{(m, r)}^{-}, \alpha_{(m, r)}^{+}: \tilde{\mathscr{U}}_{(m, r)} \rightarrow \mathbf{R}$ as $\mathscr{U}_{l}$ and $\alpha_{l}^{-}, \alpha_{l}^{+}$, where $1 \leq l \leq N$. Without loss of generality we may assume that the family $\left(\mathscr{U}_{l}\right)_{1 \leq l \leq N}$ forms a semialgebraic partition of $p_{i}(W)$.

Finally, let us consider, for $1 \leq l \leq N$, the semialgebraic sets defined by the formulas

$$
\begin{aligned}
\mathscr{T}_{l}:= & \left\{\left(x_{1}, \ldots, x_{i+1}\right) \in \mathbf{R}^{i+1} ;\left(x_{1}, \ldots, x_{i}\right) \in \mathscr{U}_{l}, \alpha_{l}^{-}\left(x_{1}, \ldots, x_{i}\right)\right. \\
& \left.\leq x_{i+1} \leq \alpha_{l}^{+}\left(x_{1}, \ldots, x_{i}\right)\right\} .
\end{aligned}
$$

Our construction guarantees that, for $\mathscr{U}_{l}=\tilde{\mathscr{U}}_{(m, r)}$, the set $\mathscr{T}_{l}$ is contained in $\bar{T}_{m}$ and that the union of all sets $\mathscr{T}_{l}$ covers $p_{i+1}(W)$ (in fact they form a partition of $\left.p_{i+1}(W)\right)$.

Moreover, the sets $\mathscr{T}_{l}$ satisfy condition (ii) of Proposition 1 above.

The continuous semialgebraic functions $\eta_{(l, j)}: \mathscr{T}_{l} \rightarrow \mathbf{R}^{n-i-1}$ are obtained by embedding $\mathscr{T}_{l}$ in a suitable semialgebraic set $\bar{T}_{m}$ and restricting the functions $\xi_{(m, s)}: \bar{T}_{m} \rightarrow \mathbf{R}^{n-i-1}$ to $\mathscr{T}_{1}$. Therefore conditions (i) and (iii) of the proposition are also satisfied.

With the same notations and assumptions as before, we make the following remarks:

Remark 2. Our construction of the semialgebraic sets and functions $\mathscr{T}_{l}, \mathscr{U}_{l}, \alpha_{l}^{+}$, $\alpha_{l}^{-}$implies that, for any $z \in \mathscr{U}_{l}$, the following equality holds:

$$
\{z\} \times\left[\alpha_{l}^{-}(z), \alpha_{l}^{+}(z)\right]=\left(\mathscr{T}_{l}\right)_{z}
$$

This is an immediate consequence of formula (*) in the proof of Proposition 1. 
Remark 3. Our algorithms operate on formulas of the first-order language $\mathscr{L}(A)$ with the aim of manipulating geometrical objects. In this sense the algorithm underlying Proposition 1 transforms in admissible time the input formula defining the semialgebraic set $W$ into a family of quantifier-free formulas $\varphi_{(l, j)} \in \mathscr{L}(A)$ in $i+n$ variables $X_{1}, \ldots, X_{i}, Y_{1}, \ldots, Y_{n}$ satisfying $\sum_{(l, j)} d\left(\varphi_{(l, j)}\right)=D^{n^{(1)}}$.

The main property of these formulas $\varphi_{(l, j)}$ consists of the following: for each $z \in \mathscr{U}_{l}$, the formula $\varphi_{(l, j)}\left(z, Y_{1}, \ldots, Y_{n}\right)$ describes a semialgebraic curve contained in the fiber $W_{z}$. The parametric form of this curve is the semialgebraic continuous function $\eta_{(l, j)}$ of the proposition and $\varphi_{(l, j)}$ describes the graph of $\eta_{(l, j)}$.

In a similar way the algorithm computes in admissible time quantifier-free formulas $\Theta_{(i, j)}^{+}, \Theta_{(l, j)}^{-}$of $\mathscr{L}(A)$ satisfying $\sum_{(l, j)}\left(d\left(\Theta_{(l, j)}^{+}\right)+d\left(\Theta_{(l, j)}^{-}\right)\right)=D^{n^{o(l)}}$. These formulas describe the endpoints of the parametric curves $\eta_{(l, j)}$.

Remark 4. We observe that Proposition 1 is a generalization of Theorem 7 of [HRS2]. In fact for the case of a closed and bounded semialgebraic set this theorem follows from Proposition 1 putting $i=0$.

The next proposition represents a parametric version of the admissible curve selection lemma of Section 4 of [HRS3].

Proposition 5. Let $W \subset \mathbf{R}^{n}$ be an explicitly given semialgebraic set. For fixed $0 \leq i<n$, it is possible to construct in admissible time the following items:

- A semialgebraic subdivision $\left(\mathscr{W}_{r}\right)_{1 \leq r \leq R}$ of $W$.

- A family of continuous semialgebraic functions $f_{r}: \mathscr{W}_{r} \rightarrow \mathbf{R}_{\geq 0}$.

- Another family of continuous semialgebraic functions $g_{(r, j)}^{-}, g_{(r, j)}^{+}: V_{r} \rightarrow \mathbf{R}^{n-i}$ where $1 \leq j \leq R_{r}$ and $V_{r}$ is the semialgebraic set defined by

$$
V_{r}:=\left\{(x, t) \in \mathscr{W}_{r} \times \mathbf{R} ; 0 \leq t \leq f_{r}(x)\right\}
$$

These semialgebraic sets and functions satisfy the following conditions: let $x:=\left(x_{1}, \ldots, x_{n}\right)$ be an arbitrary point of $\mathscr{W}_{r}$ and let $t$ be an arbitrary element of $R$ such that $(x, t) \in V_{r}$ holds. Then:

- $\left(x_{1}, \ldots, x_{i}, g_{(r, j)}^{+}(x, t)\right) \in W\left(\right.$ resp. $\left.\left(x_{1}, \ldots, x_{i}, g_{(r, j)}^{-}(x, t)\right) \in W\right)$.

- $g_{(r, j)}^{+}(x, 0)=g_{(r, j)}^{-}(x, 0)=\left(x_{i+1}, \ldots, x_{n}\right)$.

- $p_{i+1}\left(x_{1}, \ldots, x_{i}, g_{(r, j)}^{+}(x, t)\right)=\left(x_{1}, \ldots, x_{i}, x_{i+1}+t\right)$ and

$$
p_{i+1}\left(x_{1}, \ldots, x_{i}, \tilde{g_{(r, j)}}(x, t)\right)=\left(x_{1}, \ldots, x_{i}, x_{i+1}-t\right)
$$

- For each $x^{\prime}:=\left(x_{1}, \ldots, x_{i+1}+t\right)\left(\right.$ resp. $\left.x^{\prime}:=\left(x_{1}, \ldots, x_{i+1}-t\right)\right)$ and each semialgebraically connected component of $W_{x^{\prime}}$, a couple of indices $(r, j)$ exists such that $\left(x_{1}, \ldots, x_{i}, g_{(r, j)}^{+}(x, t)\right)\left(\right.$ resp. $\left.\left(x_{1}, \ldots, x_{i}, g_{(r, j)}^{-}(x, t)\right)\right)$ belongs to this component. 
Proof. We describe only the construction of the functions $g_{(r, j)}^{+}$(the functions $g_{(\bar{r}, j)}^{-}$ are obtained similarly). Let $\tilde{W} \in \mathbf{R}^{2 n-i+1}$ be the semialgebraic set defined in the following way:

$$
\begin{gathered}
\left(x_{1}, \ldots, x_{n}, y_{i+1}, \ldots, y_{n}, t\right) \in \tilde{W} \Leftrightarrow\left(x_{1}, \ldots, x_{n}, x_{1}, \ldots, x_{i}, y_{i+1}, \ldots, y_{n}\right) \in W \times W, \\
y_{i+1}=x_{i+1}+t, \quad 0 \leq \sum_{i+1 \leq l \leq n}\left(x_{l}-y_{l}\right)^{2} \leq 2 t^{2}, \quad t \geq 0 .
\end{gathered}
$$

Let $p: \mathbf{R}^{2 n-i+1} \rightarrow \mathbf{R}^{n+1}$ be the canonical projection which forgets the last $n-i$ coordinates $y_{i+1}, \ldots, y_{n}$ and let $q: \mathbf{R}^{n+1} \rightarrow \mathbf{R}^{n}$ be the one which forgets the last coordinate $t$. Observe that $W=(q \circ p)(\tilde{W})$ holds.

In the same way as in the proof of Proposition 1, we obtain a semialgebraic subdivision $\left(\mathscr{W}_{r}\right)_{1 \leq r \leq R}$ of $W=(q \circ p)(\tilde{W})$ and a semialgebraic subdivision $\left(\mathscr{H}_{r}\right)_{1 \leq r \leq R}$ of $p(\tilde{W})$ such that, for any $x \in W$, the fiber $\left(\mathscr{H}_{r}\right)_{x}$ is a subinterval of the half-line $t \geq 0$ containing 0 and with a right endpoint $h_{r}(x) \geq 0$ which may be infinite. We do not repeat the proof of this fact which is the same as the corresponding arguments in Proposition 1. We remark only that attention has to be paid to our weaker assumptions on $W$, which is no longer assumed to be closed and bounded. (This makes it necessary also to admit the value $\infty$ for $h_{r}$.)

We define $f_{r}(x):=\frac{1}{2} h_{r}(x)$ if $h_{r}(x)$ is finite, and $f_{r}(x):=1$ if $h_{r}(x)=+\infty$.

The continuous semialgebraic maps $g_{(r, j)}$ are constructed in the same way as the functions $\eta_{(l, j)}$ in the proof of Proposition 1. We do not repeat the arguments.

With the same notations and assumptions as in Proposition 5, we have

Remark 6. Each of the semialgebraic continuous functions $g_{(r, j)}^{+}: V_{r} \rightarrow \mathbf{R}^{n-i}$ in Proposition 5 is given by a quantifier-free formula $\Gamma_{(r, j)}^{+}$which belongs to $\mathscr{L}(\mathbf{A})$ and depends on the variables $X_{1}, \ldots, X_{n}, T, Y_{i+1}, \ldots, Y_{n}$. The set of these formulas is obtained in admissible time from the quantifier-free input formula defining $W$.

The continuous semialgebraic function $g_{(r, j)}^{+}$parametrizes, for each $x \in \mathscr{W}_{r}$, a closed and semialgebraically connected curve in the fiber $W_{p_{1}(x)}$ passing through $x$.

All these curves (which depend on the parameter $x$ ) are defined uniformly by a quantifier-free formula $\Psi_{(r, j)}^{+} \in \mathscr{L}(\mathbf{A})$ in the variables $X_{1}, \ldots, X_{n}, Y_{1}, \ldots, Y_{n}$ which we obtain in admissible time by eliminating the existential quantifier in the formula

$$
\left(\left(X_{1}, \ldots, X_{n}, T, Y_{i+1}, \ldots, Y_{n}\right) \in \operatorname{Graph}\left(g_{(r, j)}^{+}\right) \wedge X_{1}=Y_{1} \wedge \cdots \wedge X_{i}=Y_{i}\right)
$$

In the same way we obtain, for each $r, j$, a quantifier-free formula $\Psi_{(\mathbf{r}, j)}^{-} \in \mathscr{L}(\mathbf{A})$ in the variables $X_{1}, \ldots, X_{n}, Y_{1}, \ldots, Y_{n}$ which defines uniformly all the curves given by $g_{(r, j)}^{-}$.

In a similar way we obtain in admissible time quantifier-free formulas $\Omega_{(r, j)}^{+}$ (resp. $\Omega_{(r, j)}^{-}$) of $\mathscr{L}(\mathrm{A})$ depending on the variables $X_{1}, \ldots, X_{n}, Y_{1}, \ldots, Y_{n}$ such that, 
for each point $x=\left(x_{1}, \ldots, x_{n}\right)$ of $W, \Omega_{(r, j)}^{+}\left(x, Y_{1}, \ldots, Y_{n}\right)\left(\operatorname{resp} . \Omega_{(r, j)}^{-}\left(x, Y_{1}, \ldots, Y_{n}\right)\right)$ defines the endpoint $\left(x_{i}, \ldots, x_{i}, g_{(r, j)}^{+}(x, f(x))\right)\left(\right.$ resp. $\left.\left(x_{1}, \ldots, x_{i}, g_{(r, j)}^{-}(x, f(x))\right)\right)$.

Remark 7. In the case that $W$ is a smooth and bounded hypersurface described by a regular polynomial equation $F=0$ (i.e., $\nabla F$ vanishes nowhere in $W$ ), the functions $f_{r}, g_{(r, j)}^{+}, g_{(r, j)}^{-}$of Proposition 5 may be chosen such that the following condition is satisfied: for each $x:=\left(x_{1}, \ldots, x_{n}\right) \in W$ such that

$$
F\left(x_{1}, \ldots, x_{i}, X_{i+1}, \ldots, X_{n}\right)
$$

is a regular polynomial equation for the fiber $W_{p_{i}(x)}$ contained in $\mathbf{R}^{n-i}$, the points $\pi_{i+1}\left(x_{1}, \ldots, x_{i}, g_{(r, j)}^{+}(x, f(x))\right)$ and $\pi_{i+1}\left(x_{1}, \ldots, x_{i}, g_{(r, j)}^{-}(x, f(x))\right)$ are not critical values of the projection map $\pi_{i+1}: W_{p(x)} \rightarrow \mathbf{R}$. The proof of this refinement is essentially the same as the proof of Proposition 5. The only point to be modified is the following: in the definition of $\tilde{W}$ we have to add the semialgebraic condition:

$$
W_{p_{i}(x)} \text { smooth } \Rightarrow y_{i+1} \text { is not a critical value for }\left.\pi_{i+1}\right|_{W_{p_{i}(x)}} \text {. }
$$

Therefore in the case of a smooth and bounded hypersurface $W$ given by a regular polynomial equation we are able to move points outside from critical fibers replacing $x$ by $\left(p_{i}(x), g_{(r, j)}^{+}\left(x, f_{r}(x)\right)\right)$ and $\left(p_{i}(x), g_{(r, j)}^{-}\left(x, f_{r}(x)\right)\right)$ (compare (3) of Section 2.1).

We now give a uniform construction of $M$-functions. It is well known that after a generic linear change of coordinates, each new coordinate becomes a Morse- and consequently an $M$-function. We realize this geometrical idea by a recursive construction of generic coordinates which uses, at each step, new indeterminates for the coefficients of the linear variable transformation involved and which works in a real closed extension of the field $\mathbf{R}$.

Let $\delta_{j}^{(i)}, 1 \leq i \leq j \leq n$, be algebraically independent elements over $\mathbf{R}$, which we order in the following way:

- $\delta_{j^{\prime}}^{\left(i^{\prime}\right)}<\delta_{j}^{(i)}$ iff $i^{\prime}>i$ or $i^{\prime}=i$ and $j^{\prime}>j$.

- $0<\delta_{j^{\prime}}^{\left(i^{\prime}\right)}<z$ for every positive element $z \in \mathbf{R}\left(\delta_{1}^{(1)}, \delta_{2}^{(1)}, \ldots, \delta_{j}^{(i)}\right)$ where the variables $\delta_{1}^{(1)}, \ldots, \delta_{j}^{(i)}$ are exactly all the variables greater than $\delta_{j^{\prime}}^{\left(i^{\prime}\right)}$.

For $i, 1 \leq i \leq n$, let $\mathbf{R}_{i}$ be the real closure of the field $\mathbf{R}\left(\delta_{1}^{(1)}, \delta_{2}^{(1)}, \ldots, \delta_{n}^{(i)}\right), \mathbf{A}_{i}:=$ $\mathbf{A}\left[\delta_{1}^{(1)}, \delta_{2}^{(1)}, \ldots, \delta_{n}^{(i)}\right], \mathbf{R}^{\prime}:=\mathbf{R}_{n}$, and $\mathbf{A}^{\prime}:=\mathbf{A}_{n}$.

We consider independent linear forms $Z_{i}, 1 \leq i \leq n$, defined as follows:

$$
Z_{i}:=\delta_{i}^{(i)} X_{i}+\delta_{i+1}^{(i)} X_{i+1}+\cdots+\delta_{n}^{(i)} X_{n}
$$

For a semialgebraic set $W \subset \mathbf{R}^{n}$ defined by a formula $\Phi$ of $\mathscr{L}(\mathbf{A})$, we denote by $W^{\prime} \subset \mathbf{R}^{\prime n}$ the interpretation of $\Phi$ in $\mathbf{R}^{\prime n}$.

We conclude this section by considering the case of a smooth and bounded hypersurface. 
Suppose as before that $S$ is a bounded and smooth hypersurface of $\mathbf{R}^{n}$ given by a regular equation $F=0$, where $F$ is a polynomial of $\mathbf{A}\left[X_{1}, \ldots, X_{n}\right]$. Thus the gradient vector $\nabla F$ vanishes nowhere on $S$. Let $D:=\operatorname{deg} F$.

Lemma 8. A nonzero n-variate polynomial $P$ with coefficients in $\mathbf{A}$ having the following property exists: for any real closed extension field $L$ of $\mathbf{R}$ and for any point $\left(\alpha_{1}, \ldots, \alpha_{n}\right)$ of $L^{n} \backslash\{P=0\}$, the linear form $\alpha_{1} X_{1}+\cdots+\alpha_{n} X_{n}$ induces an $M$-projection on the hypersurface $\left\{x \in L^{n} ; F(x)=0\right\}$.

Proof (Sketch). Take the Gauss map of the hypersurface $S$ into the $n$-sphere, square its coordinates and apply the semialgebraic version of Sard's theorem [BCR, Theorem 9.5.2] to this map. Then use the Transfer Principle [BCR, Proposition 5.2.3].

Corollary 9. Fix $0 \leq i \leq n-1$. Let $\left(t_{1}, \ldots, t_{i}\right)$ be a point of $\mathbf{R}_{i}^{i}$ such that $F\left(t_{1}, \ldots, t_{i}, X_{i+1}, \ldots, X_{n}\right)=0$ is a regular equation for the fiber $S_{\left(t_{1}, \ldots, t_{1}\right)}^{\prime}$ contained in $\mathbf{R}^{\prime n-i}$. Observe that $S_{\left(t_{1}, \ldots, t_{1}\right)}^{\prime}$ is a smooth hypersurface. Then the linear form $Z_{i+1}:=\delta_{i+1}^{(i+1)} X_{i+1}+\delta_{i+2}^{(i+1)} X_{i+2}+\cdots+\delta_{n}^{(i+1)} X_{n}$ induces an $M$-projection on $S_{\left(i_{1}, \ldots, t_{1}\right)}^{\prime}$

Proof. Let $P$ be the polynomial of Lemma 8 corresponding to the hypersurface $S_{\left(t_{1}, \ldots, t_{i}\right)}$ of the affine space $\mathbf{R}_{i}^{n-i}$. The coefficients of $P$ are elements of $\mathbf{R}_{i}$. Thus we have $P\left(\delta_{i+1}^{(i+1)}, \delta_{i+2}^{(i+1)}, \ldots, \delta_{n}^{(i+1)}\right) \neq 0$ since the elements $\delta_{i+1}^{(i+1)}, \ldots, \delta_{n}^{(i+1)}$ are algebraically independent over $\mathbf{R}_{i}$. This implies that the linear form $Z_{i+1}$ induces an $M$-projection on $S_{\left(t_{1}, \ldots, t_{i}\right)}^{\prime}$.

In the situation of Corollary 9 we write $Z_{i+1}$ for the $M$-projection induced by the linear form $Z_{i+1}$ on $S_{\left(t_{1}, \ldots, t_{1}\right)}^{\prime}$.

Remark 10. Corollary 9 implies that the linear form $Z_{1}$ induces an $M$-projection on $S^{\prime}$ which is denoted by $Z_{1}$.

The curves which occur when we apply the roadmap construction [HRS3, Section 4] to $S^{\prime}$ and to the $M$-projection $Z_{1}$ have endpoints with coordinates in $\mathbf{R}_{1}$ because $Z_{1}$ is a linear form of $\mathbf{R}_{1}\left[X_{1}, \ldots, X_{n}\right]$. Therefore the $Z_{1}$-images of these points also belong to $\mathbf{R}_{1}$. If $t \in \mathbf{R}_{1}$ is a regular (i.e., not a critical) value of $Z_{1}$, the semialgebraic set $Z_{1}^{-1}(t) \cap S^{\prime}$ is a smooth variety and $Z_{2}$ is an $M$-projection of $Z_{1}^{-1}(t) \cap S^{\prime}$ following Corollary 9. Iterating this argument we see that $Z_{1}$, $Z_{2}, \ldots, Z_{n-1}$ are $M$-projections on all the fibers which occur in the construction [HRS3] of a roadmap of $S^{\prime}$ passing through a given point $x$ of $S$. This observation remains true when the point $x$ belongs to $S^{\prime} \cap \mathbf{R}_{1}^{n}$.

We denote by $\sigma$ the roadmap of $S$ given by the construction in [HRS3]. This roadmap is defined by a quantifier-free formula $\Sigma$ of $\mathscr{L}(\mathbf{A})$ which we obtain in admissible time from the input polynomial $F$ which represents the hypersurface $S$. Observe that $\sigma^{\prime}$ is also a roadmap of $S^{\prime}$. 
The following lemma describes a parametrized construction of a roadmap passing through a given point.

Lemma 11. As before, let $S$ be a bounded, smooth hypersurface of $\mathbf{R}^{n}$ given by a regular equation $F=0$ where $F$ is a polynomial of $\mathbf{A}\left[X_{1}, \ldots, X_{n}\right]$ and let $\sigma$ be the roadmap of $S$ introduced above. Then it is possible to construct in admissible time from the input $F$ a quantifier-free formula $\Phi$ in $2 n$ variables $X:=\left(X_{1}, \ldots, X_{n}\right)$, $Y:=\left(Y_{1}, \ldots, Y_{n}\right)$ which belongs to $\mathscr{L}\left(\mathbf{A}^{\prime}\right)$ and which verifies the following condition: for each $x \in S$, the formula $\Phi(x, Y)$ describes a roadmap $\sigma_{x}^{\prime}$ of $S^{\prime}$ which contains the point $x$ and the curve $\sigma^{\prime}$ in such a way that $\sigma^{\prime}$ also forms a roadmap of $\sigma_{x}^{\prime}$. The algorithm presents $\Phi$ in a disjunctive form as $\Phi=\bigvee_{1 \leq s \leq N} \Phi_{s}$ such that, for each $x^{\prime} \in S^{\prime}$, the formula $\Phi_{s}\left(x^{\prime}, Y\right)$ describes a point or a closed semialgebraically connected curve contained in $S^{\prime}$. Moreover, we have $d(\Phi)=D^{n^{(1)}}$.

Proof. Our procedure is a modified version of the roadmap construction of Section 4 of [HRS3], which applied to $S^{\prime}$ produces in admissible time three item classes of the following type:

$M$-directions for $S^{\prime}$.

Continuous semialgebraic curves contained in $S^{\prime}$.

Base points obtained intersecting the curves above with suitable fibers of $M$-directions.

We indicate only the modifications which the algorithm of [HRS3] that produces these item classes undergoes in order to construct the output formula $\Phi$ in the statement above. The first modification concerns the choice of $M$-directions. The new $M$-directions are projection maps induced on $S^{\prime}$ and fibers of $S^{\prime}$ by the linear forms $Z_{i}:=\delta_{i}^{(i)} X_{i}+\delta_{i+1}^{(i)} X_{i+1}+\cdots+\delta_{n}^{(i)} X_{n}$ where $1 \leq i \leq n$. According to Remark 10 we may suppose that the roadmap construction of [HRS3] uses only these linear transformations as $M$-directions. In this sense we may think that the selection of $M$-directions is done uniformly.

Moreover, we choose the continuous semialgebraic curves of $S^{\prime}$ corresponding to the second item class above applying Propositions 1 and 5. Only the third item class requires a more careful analysis. We now suppose that the base points corresponding to this item class are already given by the algorithm of [HRS3] and we show that in fact this procedure is uniform. The rest of the proof is devoted to this question.

The coefficients of the linear forms $Z_{1}, \ldots, Z_{n}$ describe a nonsingular linear transformation (over $\mathbf{A}^{\prime}$ ) of the variables $X_{1}, \ldots, X_{n}$. Thus we may replace the variables $X_{1}, \ldots, X_{n}$ by $Z_{1}, \ldots, Z_{n}$. This substitution transforms the polynomial $F$ to another one which depends on the new variables $Z_{1}, \ldots, Z_{n}$. The coefficients of the new polynomial belong to the field of fractions of $\mathbf{A}^{\prime}$. Multiplying this polynomial by a suitable nonzero element of $\mathbf{A}^{\prime}$ (in fact the determinant of an $n \times n$ matrix given by the coefficients of the forms $Z_{1}, \ldots, Z_{n-i}$ ) we cancel denominators and thus obtain a polynomial $G$ with coefficients in $\mathbf{A}^{\prime}$.

Obviously this procedure is completely algorithmic, can be performed in 
admissible time, and the new polynomial $G$ satisfies $\operatorname{deg} G=\operatorname{deg} F$. Moreover, $G$ is a regular equation of the smooth hypersurface $S^{\prime}$.

Applying Propositions 1 and 5 to the semialgebraic set $S^{\prime}$ given by the regular equation $G=0$ we obtain in admissible time for each $i, 0 \leq i \leq n-1$, quantifierfree formulas of degree $D^{n^{(\prime)}}$ of $\mathscr{L}\left(\mathbf{A}^{\prime}\right)$, any of which describes a parametrized family of semialgebraically connected curves lying in $S^{\prime}$. The parameters on which these families depend range over suitable semialgebraic subsets of $\mathbf{R}^{\prime i}$ (compare Remarks 3 and 6). We write $\left(\Psi_{j}^{(i)}\right)_{0 \leq j \leq M_{i}}$ for the family of these formulas.

The quantifier-free formulas $\Psi_{j}^{(i)}$ of $\mathscr{L}\left(\mathbf{A}^{\prime}\right)$ involve the $2 n$ variables $Z_{1}, \ldots, Z_{n}$, $U_{1}, \ldots, U_{n}$. Let $Z:=\left(Z_{1}, \ldots, Z_{n}\right)$ and $U:=\left(U_{1}, \ldots, U_{n}\right)$.

Analyzing the roadmap construction of [HRS3] we see that this algorithm constructs implicitly a family $q_{j h}^{(i)}\left(0 \leq i \leq n-1,0 \leq j \leq M_{i}, 0 \leq h \leq H_{i}\right)$ of continuous semialgebraic functions with domains contained in $S^{\prime}$ and values in $\mathbf{R}^{\prime}$. These functions describe the coordinates of the base points obtained by the procedure of [HRS3].

Let us consider the following expression $\Xi$ which depends on the two sets of variables $Z:=\left(Z_{1}, \ldots, Z_{n}\right)$ and $U:=\left(U_{1}, \ldots, U_{n}\right)$ :

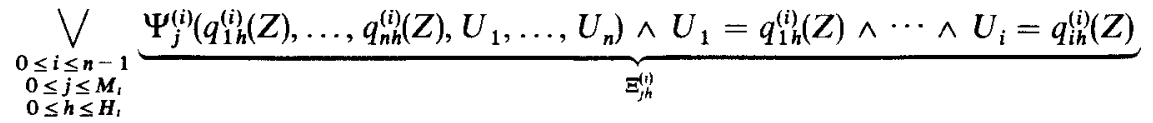

We denote the conjunctions appearing in the expression $\Xi$ above by $\Xi_{j h}^{(i)}$. Observe that Remarks 3 and 6 guarantee that, for any point $z$ of $S^{\prime}$, the formula $\Xi_{j h}^{(i)}(z, U)$ defines a point of $S^{\prime}$ or a semialgebraically connected curve which is contained in $S^{\prime}$.

Let $\mathfrak{I}$ be the regular $n \times n$ matrix defined by the coefficients of the linear forms $Z_{1}, \ldots, Z_{n}$ and let $\mathfrak{I}^{-1}$ be its inverse. Transforming the variables $Z_{1}, \ldots, Z_{n}$ by $\mathfrak{J}^{-1}$ we reobtain the variables $X_{1}, \ldots, X_{n}$. Analogously let the variables $Y_{1}, \ldots, Y_{n}$ be defined transforming $U_{1}, \ldots, U_{n}$ by means of $\mathfrak{I}^{-1}$.

We denote by $\mathfrak{I}(S)$ the subset of $\mathbf{R}^{\prime \prime}$ obtained transforming the semialgebraic set $S$ by means of the matrix $\mathfrak{I}$ (observe that the set $\mathfrak{I}(S)$ is not semialgebraic).

Let $z$ be a point of $\mathfrak{I}(S)$.

From the roadmap construction of [HRS3] and Remarks 3, 7, and 10 we infer that the set $\mathfrak{R}(z) \subset \mathbf{R}^{\prime n}$ defined by $\Xi(z, U)$ is a roadmap of $S^{\prime}$ which contains the point $z$.

We now apply the algorithm of [HRS3] to the hypersurface $S^{\prime}$ in the following way: every time the algorithm requires an $M$-direction we choose one of the projection maps $Z_{1}, \ldots, Z_{n}$ according to Remark 10 . In this manner we obtain a roadmap $\sigma_{1}$ of $S^{\prime}$. From the previous observations we deduce that $\sigma_{1}$ is contained in the curve $\mathfrak{R}(z)$ for any point $z$ of $\mathfrak{I}(S)$. Without loss of generality we may suppose that $\mathfrak{I}(\sigma)$ is also contained in $\mathfrak{R}(z)$ for all $z \in \mathfrak{I}(S)$.

Unfortunately the roadmap construction of [HRS3], as far as the variables $Z_{1}, \ldots, Z_{n}$ are concerned, does not present the expressions $\Xi_{j h}^{(i)}$ as a quantifier-free formula of degree $D^{n^{\text {(1) }}}$.

Suppose for the moment that this is the case, i.e., that the expression $\Xi_{j h}^{(i)}$ is 
represented by a quantifier-free formula of $\mathscr{L}\left(\mathbf{A}^{\prime}\right)$ which can be obtained in admissible time and which has degree $D^{n^{O(1)}}$. Then we have $N=D^{n^{011}}$ such formulas which depend on the variables $Z_{1}, \ldots, Z_{n}$ and $U_{1}, \ldots, U_{n}$. Transforming these variables by means of the matrix $\mathfrak{I}^{-1}$ we obtain quantifier-free formulas $\Phi_{s}$ in the variables $X_{1}, \ldots, X_{n}$ and $Y_{1}, \ldots, Y_{n}$ where $1 \leq s \leq N$.

Since for any $z \in S^{\prime}$ the expressions $\Xi_{j h}^{(i)}(z, U)$ define points of $S^{\prime}$ or semialgebraically connected curves contained in $S^{\prime}$, the same property is shared by the formula $\Phi_{s}(x, Y)$ for any $x \in S^{\prime}$ and any $1 \leq s \leq N$. Under the hypothesis made before we are able to construct the formulas $\Phi_{s}$ in admissible time and we have $d\left(\Phi_{s}\right)=D^{n^{o(i)}}$. The formulas $\Phi_{s}$ and $\Phi:=\bigvee_{1 \leq s \leq N} \Phi_{s}$ therefore satisfy the requirements of the lemma if we are able to show that the expressions $\Xi_{j h}^{(i)}(Z, U)$ are represented by quantifier-free formulas of $\mathscr{L}\left(\mathbf{A}^{\prime}\right)$ which can be obtained from the input polynomial $F$ in admissible time.

From the construction in [HRS3] it is easy to see that the number $H_{i}$ of base points in the $i$ th step of the algorithm and the number $M_{i}$ of formulas $\Psi_{j}^{(i)}$ are of order $D^{n^{o(1)}}$ (recall that $H_{i}$ and $M_{i}$ are quantities which were introduced in the expression $\Xi$ above).

We finish the proof representing each expression $\Xi_{j h}^{(i)}$ by a quantifier-free formula of degree $D^{n^{a(1)}}$ which can be obtained in admissible time.

For this purpose, we apply the quantifier-elimination algorithm of [HRS2] or [R3] to the purely existential prenex formulas $\Lambda_{j h}^{(i)}$ which we introduce below. These formulas have degree $D^{n^{o(1)}}$ and contain not more than $3 n^{2}+2 n$ variables.

For the moment let $i, j$, and $h$ be fixed.

From Remarks 3 and 6 we obtain quantifier-free formulas $\Theta_{l}^{(i)}$ and $\Omega_{l}^{(i)}$ in not more than $2 n$ variables which describe the endpoints of the parametrized family of curves given by the formula $\Psi_{j}^{(i)}$.

The following prenex formulas describe all possible intersections of the parametrized families of curves defined by the formulas $\Phi_{j}^{(i)}$, where $0 \leq j \leq M_{i}$, with fibers which contain an endpoint of one of these curves. The following two formulas correspond to $\Psi_{j}^{(i-1)}$ :

$$
\left(\exists V_{i+1} \cdots \exists V_{n}\right) \quad\left(\Psi_{j}^{(i-1)}(Z, U) \wedge \Theta_{l}^{(i-1)}\left(Z_{1}, \ldots, Z_{i}, U_{1}, \ldots, U_{i}, V_{i+1}, \ldots, V_{n}\right)\right)
$$

and

$$
\left(\exists V_{i+1} \cdots \exists V_{n}\right) \quad\left(\Psi_{j}^{(i-1)}(Z, U) \wedge \Omega_{l}^{(i-1)}\left(Z, U_{1}, \ldots, U_{i}, V_{i+1}, \ldots, V_{n}\right)\right)
$$

All these prenex formulas (with $0 \leq j \leq M_{i}$ ) contain only one block of existential quantifiers and applying the quantifier-elimination algorithm of [HRS2] or [R3] to them we obtain in admissible time a family of equivalent quantifier-free formulas $\left(\Gamma_{\ell}^{(i)}\right)_{0 \leq \ell \leq L_{1}}$ of degree $D^{n^{\alpha(1)}}$ which depend on the variables $Z_{1}, \ldots, Z_{n}, U_{1}, \ldots, U_{n}$.

We introduce new variables $T_{t}^{(s)}$, where $0 \leq s \leq i-1$ and $1 \leq t \leq n$, $W_{t^{\prime}}^{\left(s^{\prime}\right)}$, where $1 \leq s^{\prime} \leq i-1$ and $1 \leq t^{\prime} \leq n$, and $V_{v}^{(p)}$, where $1 \leq p \leq i-1$ and $p<r \leq n$. Observe that the number of these variables does not exceed $3 n^{2}$. 
We now define, for each triple of indices $i, j, h$, a purely existential prenex formula $\Lambda_{j h}^{(i)}$ which has degree $D^{n^{O(1)}}$ and can be constructed in admissible time. This formula describes a parametrized family of continuous semialgebraic curves which connects base points lying in the same semialgebraically connected component of $S^{\prime}$. The formula $\Lambda_{j h}^{(i)}$ is given in the way we indicate below.

Let $[\exists]$ be the existential quantifier block

$$
\left(\exists T_{t}^{(s)}\right)\left(\exists W_{i^{\prime}}^{\left(s^{\prime}\right)}\right)\left(\exists V_{r}^{(p)}\right),
$$

where $0 \leq s \leq i-1,1 \leq t \leq n, 1 \leq s^{\prime} \leq i-1,1 \leq t^{\prime} \leq n, 1 \leq p \leq i-1, p<r \leq n$.

Furthermore, let $[\star]$ be the following quantifier-free formula:

$$
\begin{aligned}
\Omega_{l_{0}}^{(0)}\left(Z, T^{(0)}\right) & \wedge \Gamma_{\ell_{1}}^{(1)}\left(T_{1}^{(0)}, V^{(1)}, W^{(1)}\right) \wedge \Omega_{l_{1}}^{(1)}\left(W^{(1)}, T^{(1)}\right) \\
& \wedge \Gamma_{\ell_{2}}^{(2)}\left(T_{1}^{(0)}, T_{2}^{(1)}, V^{(2)}, W^{(2)}\right) \wedge \Omega_{l_{2}}^{(2)}\left(W^{(2)}, T^{(2)}\right) \\
& \wedge \cdots \wedge \Gamma_{\ell_{1-1}}^{(i-1)}\left(T_{1}^{(0)}, \ldots, T_{i-1}^{(i-2)}, V^{(i-1)}, W^{(i-1)}\right) \wedge \Omega_{l_{l-1}}^{(i-1)}\left(W^{(i-1)}, T^{(i-1)}\right)
\end{aligned}
$$

(The formulas $\Gamma_{\ell_{k}}^{(k)}$ contained in $[\star]$ describe the coordinates of the base points which our modified version of the algorithm of [HRS3] constructs recursively, and the formulas $\Omega_{l_{k}}^{(k)}$ move these base points into noncritical fibers with respect to the projection maps $Z_{k}$.)

Finally, let $\Lambda_{j h}^{(i)}$ be the formula given in the following syntactical way:

$$
\text { [ヨ] }
$$

$$
\begin{aligned}
& \left(\Psi_{j}^{(i)}\left(T_{1}^{(0)}, \ldots, T_{i}^{(i-1)}, T_{i+1}^{(i-1)}, \ldots, T_{n}^{(i-1)}, U\right)\right. \\
& \left.\wedge U_{1}=T_{1}^{(0)} \wedge \cdots \wedge U_{i}=T_{i}^{(i-1)} \wedge[\star]\right)
\end{aligned}
$$

The quantifier-free formulas representing the expressions $\Xi_{j h}^{(i)}$ are now obtained by applying the quantifier-elimination procedure of [HRS2] or [R3] to the purely existential prenex formulas $\Lambda_{j h}^{(i)}$. It is evident that all formulas occurring in our construction are of degree $D^{n^{o(1)}}$, that there are only $D^{n^{(1)}}$ many of them, and that they can be produced in admissible time. This finishes the proof of this lemma.

Remark 12. A key point in the proof of the previous technical lemma is the simple observation that the composition of several semialgebraic functions can be described by an elementary prenex formula which is purely existential and contains therefore just one single block of quantifiers. This block can be eliminated in admissible time by means of the algorithms of [HRS2] or [R3].

\section{Description of the Connected Components in Admissible Time}

In this section we finish the proof of our main theorem: the description of the semialgebraically connected components of an explicitly given semialgebraic set in admissible time. 
Proof of the Main Theorem. According to Section 4 of [HRS4] (or Sections 2 and 3 of [CGV]) it suffices to consider the case of a smooth and bounded hypersurface $S$ of $\mathbf{R}^{n}$ given by a regular equation $F=0$ where $F$ is a polynomial of $\mathbf{A}\left[X_{1}, \ldots, X_{n}\right]$ of degree $D$.

From Lemma 11 we deduce that it is possible to construct in admissible time a quantifier-free formula $\Phi$ of $\mathscr{L}\left(\mathbf{A}^{\prime}\right)$ of degree $D^{n^{\text {O11 }}}$ which has the form of a disjunction $\bigvee_{1 \leq s \leq N} \Phi_{s}$ and in which each $\Phi_{s}$ describes a parametrized family of continuous semialgebraic curves contained in $S^{\prime}$.

The formula $\Phi$ depends on the variables $X:=\left(X_{1}, \ldots, X_{n}\right)$ and $Y:=\left(Y_{1}, \ldots, Y_{n}\right)$ and, for each point $x \in S$, the expression $\Phi(x, Y)$ defines a roadmap of $S^{\prime}$ which passes through $x$.

For each couple $s, t$, where $1 \leq s \leq t \leq N$, let $\Phi_{s t}$ be the quantifier-free formula of $\mathscr{L}\left(\mathbf{A}^{\prime}\right)$ which we obtain by applying the quantifier-elimination procedure of [HRS2] or [R3] to the formula

$$
\left(\exists Y_{1} \cdots \exists Y_{n}\right) \quad\left(\Phi_{s}\left(X, Y_{1}, \ldots, Y_{n}\right) \wedge \Phi_{t}\left(X, Y_{1}, \ldots, Y_{n}\right)\right) .
$$

Let $\mathscr{F}$ be the set of all polynomials of $\mathbf{A}^{\prime}\left[X_{1}, \ldots, X_{n}\right]$ which occur as terms in the formulas $\Phi_{s t}$. Obviously the set $\mathscr{F}$ can be constructed in admissible time and satisfies the estimate $\sum_{F \in \mathscr{F}} \operatorname{deg} F=\sum_{s, t} d\left(\Phi_{s t}\right)=D^{n^{O(1)}}$.

A (nonempty) semialgebraic subset of $\mathbf{R}^{\prime \prime}$ defined by a consistent sign condition on the elements of $\mathscr{F}$ is called an $\mathscr{F}$-cell. According to [G2] (see also [HRS1] and [HRS2]) the number of $\mathscr{F}$-cells is of order $D^{n^{\alpha(1)}}$ and we can find the consistent sign conditions defining them in admissible time.

We consider all possible nonempty intersections $V_{1}, \ldots, V_{M}$ of $\mathscr{F}$-cells with the hypersurface $S^{\prime}$. Thus we have $S^{\prime}=\bigcup_{1 \leq k \leq M} V_{k}$ and $M=D^{n^{O(1)}}$. Observe that each formula $\Phi_{s t}$ has a constant truth value on each set $V_{k}$ since the polynomials appearing as terms in $\Phi_{s t}$ belong to $\mathscr{F}$ and therefore have a constant sign on $V_{k}$.

Let $k$ with $1 \leq k \leq M$ be fixed. We consider the binary relation $\sim_{k}$ on the set $\{1,2, \ldots, M\}$ defined as follows:

for $s, t \in\{1,2, \ldots, M\}$ let $s \sim_{k} t$ hold if and only if the formula $\Phi_{s t}$ is true on $V_{k}$.

The relation $\sim_{k}$ is symmetric but not necessarily reflexive. Let $\mathscr{M}_{k}$ be its domain which is contained in $\{1,2, \ldots, M\}$. Then the transitive closure of the relation $\sim_{k}$ induces an equivalence relation on the set $\mathscr{M}_{k}$.

Let $\Lambda_{1}, \ldots, \Lambda_{r}$ be the partition of $\mathscr{M}_{k}$ which corresponds to this equivalence relation. Computing the transitive closure of the relation $\sim_{k}$ by the algorithm of [M, Theorem V.5.3], we obtain in admissible time the partition sets $\Lambda_{1}, \ldots, \Lambda_{r}$.

For any index $p$, with $1 \leq p \leq r$, let $\Delta_{p}^{(k)}$ be the following quantifier-free formula of $\mathscr{L}\left(\mathbf{A}^{\prime}\right)$ :

$$
\bigvee_{s \in \Lambda_{p}} \Phi_{s}(X, Y)
$$

Obviously the formulas $\Delta_{p}^{(k)}$ have degree $D^{n^{(1)}}$ and can be constructed in admissible time. 
Claim. For any point $x \in V_{k} \cap S$ the formula $\Delta_{p}^{(k)}(x, Y)$ defines a semialgebraically connected component of the roadmap $\sigma_{x}^{\prime}$ introduced in Lemma 11.

Proof of the Claim. Let $x \in S \cap V_{k}$ be given and suppose that the set $\Lambda_{p}$ can be written as $\Lambda_{p}=\left\{s_{1}, \ldots, s_{\ell}\right\}$ such that $s_{j} \sim_{k} s_{j+1}$ holds for $1 \leq j \leq \ell-1$. We consider the set $\tau_{p}:=\left\{y \in \mathbf{R}^{\prime n} ; \Delta_{p}^{(k)}(x, y)\right.$ is true $\}$. We show that $\tau_{p}$ is a semialgebraically connected curve contained in $S^{\prime}$.

From the fact that $\Delta_{p}^{(k)}(x, Y)$ is identical with the formula $\bigvee_{1 \leq j \leq \ell} \Phi_{s}(x, Y)$, that the point $x$ belongs to $V_{k}$, and that $s_{1} \sim_{k} s_{2} \sim_{k} \cdots \sim_{k} s_{\ell-1} \sim_{k} s_{\ell}$ holds, we infer that there exists, for each $1 \leq j \leq \ell-1$, an element $y_{j}$ of $S^{\prime}$ which verifies the expression $\Phi_{s_{j}}\left(x, h_{j}\right) \wedge \Phi_{s_{j+1}}\left(x, y_{j}\right)$. Hence, by Lemma 11 , for each $1 \leq j \leq \ell-1$, the formula $\Phi_{s_{1}}(x, Y) \wedge \Phi_{s_{j+1}}(x, Y)$ defines a semialgebraically connected curve contained in $S^{\prime}$. This implies that $\tau_{p}$ itself is a semialgebraically connected curve contained in $S^{\prime}$.

Now consider $1 \leq p^{\prime}, p \leq r$ with $\tau_{p} \cap \tau_{p^{\prime}} \neq \varnothing$. Let $y$ be an element of $\tau_{p} \cap \tau_{p^{\prime}}$. There exist indices $s \in \Lambda_{p}$ and $t \in \Lambda_{p}$, such that $\Phi_{s}(x, y) \wedge \Phi_{t}(x, y)$ holds, whence $s \sim_{k} t$ and finally $p=p^{\prime}$. Therefore the semialgebraically connected curves $\tau_{p}$ are mutually disjoint.

Since the point $x$ belongs to $V_{k}$ the formula $\Phi(x, Y)$ is equivalent to

$$
\bigvee_{1 \leq p \leq r} \Delta_{p}^{(k)}(x, Y)
$$

and thus we obtain

$$
\sigma_{x}^{\prime}=\bigcup_{1 \leq p \leq r} \tau_{p}
$$

This finishes the proof of the claim.

Note that, in virtue of the claim above, the number $r$ of formulas $\Delta_{p}^{(k)}$ coincides with the number of semialgebraically connected components of $S$ and $S^{\prime}$ which is of order $D^{\left.n^{\alpha 11}\right)}$.

Let $\sigma^{\prime}$ be the roadmap of $S^{\prime}$ which we have already considered in Lemma 11. In view of Proposition 2 of [HRS4], we may suppose that $\sigma^{\prime}$ is given as a disjunction of quantifier-free formulas $\Sigma_{1}, \ldots, \Sigma_{r}$ of $\mathscr{L}\left(\mathbf{A}^{\prime}\right)$ of degree $D^{n^{o(1)}}$ which describe the semialgebraically connected components of $\sigma^{\prime}$.

Recall from Lemma 11 that, for each $x$ in $S$, the roadmap $\sigma_{x}^{\prime}$ of $S^{\prime}$ contains the curve $\sigma^{\prime}$.

Let $1 \leq p \leq r$. For any $1 \leq k \leq M$, and $1 \leq p^{\prime} \leq r$, and any point $x$ of $S$, the formula

$$
\left(\exists Y_{1} \cdots \exists Y_{n}\right) \quad\left(\Sigma_{p}\left(Y_{1}, \ldots, Y_{n}\right) \wedge \Delta_{p^{\prime}}^{(k)}\left(x, Y_{1}, \ldots, Y_{n}\right) \wedge \Delta_{p^{\prime}}^{(k)}(x, x)\right)
$$

says that $x$ is a point of $V_{k}$ which belongs to the $p$ th semialgebraically connected component of $S^{\prime}$, i.e., which belongs to the semialgebraically connected component 
that contains the curve defined by $\Sigma_{p}$. Therefore the formula

$$
(F=0) \wedge\left(\exists Y_{1} \cdots \exists y_{n}\right) \underset{\substack{1 \leq k \leq M \\ 1 \leq p^{\prime} \leq r}}{\bigvee}\left(\Sigma_{p}\left(Y_{1}, \ldots, Y_{n}\right) \wedge \Delta_{p^{\prime}}^{(k)}\left(X, Y_{1}, \ldots, Y_{n}\right) \wedge \Delta_{p^{\prime}}^{(k)}(X, X)\right)
$$

when restricted to points of $\mathbf{R}^{n}$, defines the $p$ th semialgebraically connected component of $S$.

We now apply the quantifier-elimination procedure of [HRS2] or [R3] to all these formulas where $1 \leq p \leq r$. In this way we obtain, in admissible time, $r$ quantifier-free formulas $\Pi_{1}, \ldots, \Pi_{r}$ of $\mathscr{L}\left(\mathbf{A}^{\prime}\right)$ which involve the variables $X_{1}, \ldots, X_{n}$ and which, restricted to points of $\mathbf{R}^{n}$, describe the semialgebraically connected components of $S$.

Unfortunately the constants contained in these formulas belong to

$$
\mathbf{A}^{\prime}=\mathbf{A}\left[\delta_{1}^{(1)}, \delta_{2}^{(1)}, \ldots, \delta_{n}^{(n-1)}, \delta_{n}^{(n)}\right]
$$

and not to the base ring $\mathbf{A}$. To remedy this situation we now apply a method which we have already used in [HRS2].

Let $\mathscr{G}$ be the set of elements of $\mathbf{A}^{\prime}\left[X_{1}, \ldots, X_{n}\right]$ which occur as terms in the formulas $\Pi_{1}, \ldots, \Pi_{r}$. The set $\mathscr{G}$ can be constructed in admissible time, it is of cardinality $D^{n^{O(1)}}$, and its elements are polynomials of degree $D^{n^{O(1)}}$ in the indeterminates $\delta_{1}^{(1)}, \delta_{2}^{(1)}, \ldots, \delta_{n}^{(n-1)}, \delta_{n}^{(n)}, X_{1}, \ldots, X_{n}$ over the ring $\mathbf{A}$.

Let $G$ be an element of $\mathscr{G}$. We consider $G$ as a polynomial in $\delta_{1}^{(1)}, \delta_{2}^{(1)}, \ldots, \delta_{n}^{(n-1)}$, $\delta_{n}^{(n)}$ with coefficients in $\mathbf{A}\left[X_{1}, \ldots, X_{n}\right]$.

Since $\delta_{1}^{(1)}, \delta_{2}^{(1)}, \ldots, \delta_{n}^{(n-1)}, \delta_{n}^{(n)}$ are positive infinitesimals with respect to the real closed field $\mathbf{R}$, any sign condition of the type $G(x)>0, G(x)<0$, or $G(x)=0$ for a point $x$ of $\mathbf{R}^{n}$ can be expressed by a quantifier-free formula of $\mathscr{L}(\mathbf{A})$ which involves only the coefficients of the polynomial $G$ which lie in $\mathbf{A}\left[X_{1}, \ldots, X_{n}\right]$. The quantifier-free formulas of $\mathscr{L}(\mathbf{A})$ which are produced in this way when $G$ runs through the set $\mathscr{G}$ can be obtained in admissible time and their number and degrees are of order $D^{n^{\text {O(1) }} \text {. }}$

Using these formulas which belong to $\mathscr{L}(\mathbf{A})$ we may replace each atomic expression in $\Pi_{1}, \ldots, \Pi_{r}$ by a quantifier-free formula that contains only constants from A. Rewriting the formulas $\Pi_{1}, \ldots, \Pi_{r}$ in this way we obtain, in admissible time $r$, quantifier-free formulas of $\mathscr{L}(\mathbf{A})$ which have degree $D^{n^{o(1)}}$ and which are equivalent to $\Pi_{1}, \ldots, \Pi_{r}$ when restricted to points of $\mathbf{R}^{n}$. In other words these formulas define the semialgebraically connected components of the hypersurface $S$. This finishes the proof of the main theorem.

\section{References}

[BCR] Bochnak, J., Coste, M., Roy, M.-F.: Géométrie algébrique réelle. Ergebnisse der Mathematik und ihrer Grenzgebiete, 3 Folge, Band 12. Springer-Verlag, Berlin, 1987.

[Ca1] Canny, J.: Some algebraic and geometric computations in PSPACE. Proc. 20th Ann. ACM Symp. on Theory of Computing, 1988, pp. 460-467. 
[Ca2] Canny, J.: The Complexity of Robot Motion Planning. MIT Press, Cambridge, MA, 1989.

[CGV] Canny, J., Grigor'ev, D. Yu., Vorobjov, N. N. (Jr.): Finding connected components of a semialgebraic set in subexponential time. AAECC J. 2(4) (1992), 217-238.

[Co] Collins, G.: Quantifier elimination for real closed fields by cylindric algebraic decomposition. Proc. Second GI Conf. on Automata Theory and Formal Languages. Lecture Notes in Computer Science, Vol. 33. Springer-Verlag, Berlin, 1975, pp. 134-183.

[G1] von zer Gathen, J.: Parallel arithmetic computations: a survey. Proc. 13th Conf. Symp. MFCS. Lecture Notes in Computer Science, Vol. 233. Springer-Verlag, Berlin, 1986, pp. $93-112$.

[G2] Grigor'ev, D.: Complexity of deciding Tarski algebra. J. Symbolic Comput. 5 (1988), 65-108.

$\left[\mathrm{GHR}^{+}\right]$Grigor'ev, D. Yu, Heintz, J., Roy, M.-F., Solernó, P., Vorobjov, N. N. (Jr.): Comptage des composantes connexes d'un ensemble semi-algébrique en temps simplement exponentiel. C. R. Acad. Sci. Paris Sér. I 312 (1990), 879-882.

[GR] Gournay, L., Risler, J.-J.: Construction of roadmaps in semi-algebraic sets. J. Appl. Algebra Engrg. Comm. Comput. (to appear).

[GV1] Grigor'ev, D., Vorobjov, N. N. (Jr.): Solving systems of polynomial inequalities in subexponential time. J. Symbolic Comput. 5 (1988), 37-64.

[GV2] Grigor'ev, D., Vorobjov, N. N. (Jr.): Counting connected components of a semialgebraic set in subexponential time. Comput. Complexity (to appear).

[HRS1] Heintz, J., Roy, M.-F., Solernó, P.: On the complexity of semialgebraic sets. Proc. Information Processing (IFIP '89), San Francisco, 1989 (G. X. Ritter, ed.). North-Holland, Amsterdam, 1989, pp. 293-298.

[HRS2] Heintz, J., Roy, M.-F., Solernó, P.: Sur la complexité du principe de Tarski-Seidenberg. Bull. Soc. Math. France 118 (1990), 101-126.

[HRS3] Heintz, J., Roy, M.-F., Solernó, P.: Single exponential path finding in semi-algebraic sets. Part I: The case of a regular bounded hypersurface. Discrete and Applied Mathematics, Proc. 8th Internat. Conf. on Applied Algebra, Algebraic Algorithms on Error Correcting, Codes $A A E C C-8$, Tokyo, 1990 (S. Sakata, ed.). Lecture Notes on Computer Science, Vol. 508. Springer-Verlag, Berlin, 1991, pp. 180-186.

[HRS4] Heintz, J., Roy, M.-F., Solernó, P.: Single exponential path finding in semialgebraic sets. Part II: The general case. Proc. Symp. in Honour of S. Abhyankar (1990) (to appear).

[HRS5] Heintz, J., Roy, M.-F, Solernó, P.: Description des composantes connexes d'un ensemble semi-algébrique en temps simplement exponential. C. R. Acad. Sci. Paris Sér. I 313 (1991), $167-170$.

[M] Mehlhorn, K.: Data Structures and Algorithms, Vol. 2. EATCS Monographs on Theoretical Computer Science, Vol. 2, Springer-Verlag, Berlin, 1984.

[R1] Renegar, J.: On the computational complexity and geometry of the first order theory of the reals, I. J. Symbolic Comput. 13 (1992), 255-300.

[R2] Renegar, J.: On the computational complexity and geometry of the first order theory of the reals, II. J. Symbolic Comput. 13 (1992), 301-328.

[R3] Renegar, J.: On the computational complexity and geometry of the first order theory of the reals, III. J. Symbolic Comput. 13 (1992), 329-352.

[SS] Schwartz, J., Sharir, M.: On the "piano movers" problem, II. General techniques for computing topological properties of real algebraic manifolds. Adv. in Appl. Math. 4 (1983), 298-351.

[W] Wüthrich, H.: Ein Entscheidungsverfahren für die Theorie der reell-abgeschlos-senen Körper. Komplexität von Entscheidungsproblemen (E. Specker and V. Strassen, eds.). Lecture Notes in Computer Science, Vol. 43, Springer-Verlag, Berlin, 1976, pp. 138-162.

Received June 8, 1992, and in revised form January 25, 1993. 